\title{
Floquet Engineering Topological Many-Body Localized Systems
}

\author{
K. S. C. Decker, ${ }^{1}$ C. Karrasch, ${ }^{1}$ J. Eisert, ${ }^{2}$ and D. M. Kennes ${ }^{3,4}$ \\ ${ }^{1}$ Technische Universität Braunschweig, Institut für Mathematische Physik, Mendelssohnstraße 3, 38106 Braunschweig, Germany \\ ${ }^{2}$ Dahlem Center for Complex Quantum Systems and Fachbereich Physik, Freie Universität Berlin, 14195 Berlin, Germany \\ ${ }^{3}$ Institut für Theorie der Statistischen Physik, RWTH Aachen University \\ and JARA-Fundamentals of Future Information Technology, 52056 Aachen, Germany \\ ${ }^{4}$ Max Planck Institute for the Structure and Dynamics of Matter, \\ Center for Free-Electron Laser Science, 22761 Hamburg, Germany
}

(Received 19 November 2019; accepted 16 April 2020; published 11 May 2020)

\begin{abstract}
We show how second-order Floquet engineering can be employed to realize systems in which manybody localization coexists with topological properties in a driven system. This allows one to implement and dynamically control a symmetry protected topologically ordered qubit even at high energies, overcoming the roadblock that the respective states cannot be prepared as ground states of nearest-neighbor Hamiltonians. Floquet engineering-the idea that a periodically driven nonequilibrium system can effectively emulate the physics of a different Hamiltonian-is exploited to approximate an effective three-body interaction among spins in one dimension, using time-dependent two-body interactions only. In the effective system, emulated topology and disorder coexist, which provides an intriguing insight into the interplay of many-body localization that defies our standard understanding of thermodynamics and into the topological phases of matter, which are of fundamental and technological importance. We demonstrate explicitly how combining Floquet engineering, topology, and many-body localization allows one to harvest the advantages (time-dependent control, topological protection, and reduction of heating, respectively) of each of these subfields while protecting them from their disadvantages (heating, static control parameters, and strong disorder).
\end{abstract}

DOI: 10.1103/PhysRevLett.124.190601

In the longstanding quest for the practical realization of key quantum technologies such as quantum computing [1], a key goal is to fight off decoherence and manipulate quantum systems in a controlled way [2]. Several promising concepts have been proposed within the past decade and have become central research fields in the study of quantum many-body phenomena: Topological phases of matter are reflected by robust degeneracies of ground states and are signified by nonlocal order parameters $[3,4]$. Many-body localization in disordered systems defies our standard understanding of thermodynamics by breaking ergodicity and barring the system from thermalization [5-8]. By this many-body localization can extend the aforementioned topological protection to high energies $[9,10]$. Floquet engineering - the idea that a periodically driven nonequilibrium system [11,12] can effectively emulate the physics of a different Hamiltonian-allows one to realize "toy models" in real-life systems and to establish stable real-time protocols to manipulate quantum states [13].

Most of the proposals on how topology, disorder, and Floquet engineering can be exploited in the design of quantum devices [14-25] either concentrate on one or two of these phenomena separately or use special models. Floquet engineering has been suggested as a route to realizing effective topological systems [26-32], but those studies neglect two-body interactions and thus ignore the fact that a generic driven system will heat up [33]. Heating can be efficiently suppressed by many-body localization [34,35], but the topological properties of a clean system are normally lost in the presence of strong disorder [36-38].

Recently, an artificial toy model exhibiting three-body interactions was identified in which topology and manybody localization coexist instead of hampering each other [39-42]. Both phenomena conspire to provide topological protection of an edge spin degree of freedom even at high energies as the quantum system is localized for the entire eigenspectrum (in contrast to only some part of it). Three body-interactions as needed to realize such artificial systems are, however, excessively difficult to realize in a real-life Hamiltonian, but they can naturally arise in Floquet-engineered setups, as suggested in Ref. [43]. Here, we build on these ideas and extend them in four different directions: (i) We show how the toy Hamiltonian of Refs. [39,40] can be obtained by means of Floquet engineering, starting from a physical quantum spin model $t \mapsto H(t)$ with time-periodic two-body interactions; (ii) we explicitly benchmark how well the effective timeindependent Floquet Hamiltonian $H^{\text {eff }}$ describes the stroboscopic physics of $t \mapsto H(t)$; (iii) we demonstrate to what 


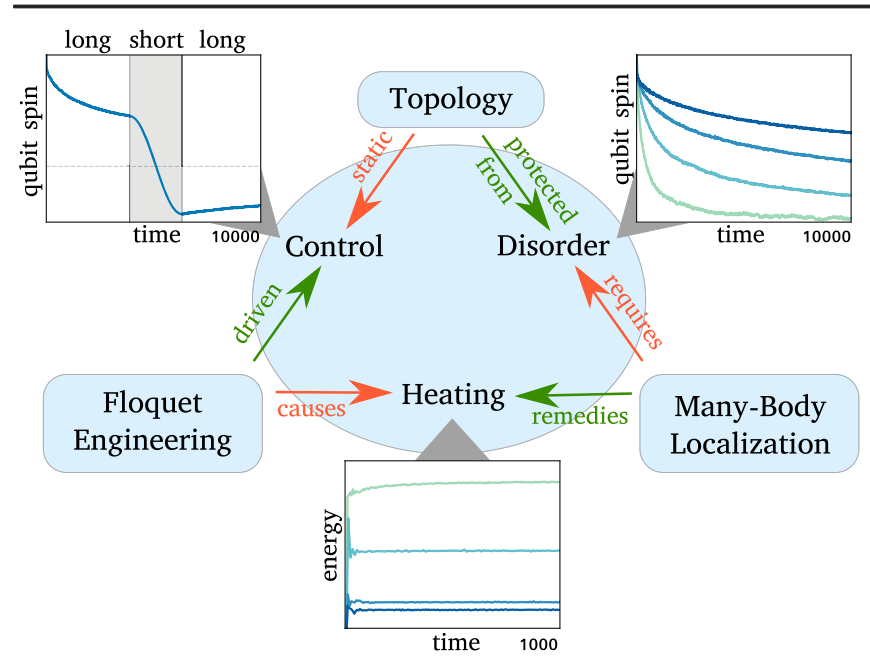

FIG. 1. We combine insights from the subfields of Floquet engineering, many-body localization, and topology. By doing so, we demonstrate how to harvest the full advantages promised by these fields-flexible control, suppression of heating, and topological protection-hile removing their respective disadvantages. These advantages are accessible with programmable quantum simulators. We explicitly illustrate this in Fig. 3 (topological protection), Fig. 4 (suppression of heating), and Fig. 5 (control), which are schematically given as insets here.

extent heating is suppressed by many-body localization; and (iv) we design a dynamical protocol that allows one to flip the edge spin on a short timescale.

In sum, Floquet engineering, many-body localization (i.e., disorder and interactions), and topological protection are three pillars on which useful quantum applications can be built. The combination of these three ingredients elegantly counteracts all their individual shortcomings-if one removes only one of them, one can no longer fully harvest their strengths. We now illustrate this explicitly and demonstrate the coexistence of topology and many-body localization in a driven system, the avoidance of heating, and the topologically protected dynamical control of quantum states. A brief summary is given in Fig. 1.

Model.-We consider a one-dimensional spin- $\frac{1}{2}$ Hamiltonian consisting of a time-periodic and a timeindependent contribution, $H(t)=H_{1}(t)+H_{2}$. The timeperiodic part is given by

$$
\begin{aligned}
H_{1}(t)= & \sqrt{\omega} \sin (\omega t) \sum_{i=1}^{L / 2} \alpha_{i} \sigma_{2 i-1}^{z} \sigma_{2 i}^{z}+\sqrt{\omega} \cos \left(\omega t+\phi_{0}\right) \\
& \times \sum_{i=1}^{(L-1) / 2}\left(\beta_{i} \sigma_{2 i}^{y} \sigma_{2 i+1}^{z}+\gamma_{i} \sigma_{2 i}^{z} \sigma_{2 i+1}^{y}\right)
\end{aligned}
$$

where $\omega$ denotes the driving frequency. The timeindependent contribution reads

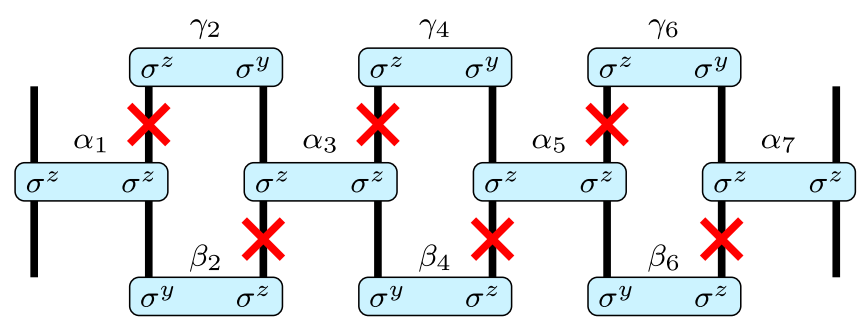

FIG. 2. Representation of the nonvanishing part of the commutator $\left[H_{1}(t), H_{1}\left(t^{\prime}\right)\right]$ relevant to the second-order Magnus expansion for a chain consisting of eight sites. The vertical lines represent the sites, while the light blue rectangles represent the terms acting on the sites. The red crosses mark terms that are commuting even though they are acting on the same site. The commutator of two two-body interactions effectively generates a three-body interaction.

$$
H_{2}=\sum_{i=1}^{L-1} V_{i} \sigma_{i}^{x} \sigma_{i+1}^{x}+\sum_{i=1}^{L} h_{i} \sigma_{i}^{x},
$$

with $\sigma_{i}^{x, y, z}$ being the Pauli matrices. The prefactors $h_{i}, V_{i}$, $\alpha_{i}, \beta_{i}, \gamma_{i}$ can be chosen differently for each lattice site $i$, and we draw them from a random Gaussian distribution with a standard deviation of $\sigma_{h, V, \alpha, \beta, \gamma}$ in order to drive the system into a many-body localized phase.

The above Hamiltonian is time periodic, $H(t+T)=$ $H(t)$, with $T=2 \pi / \omega$ for all times $t$. In order to gain some understanding of what physics one should expect to be modeled by $t \mapsto H(t)$, one can exploit the fact that the stroboscopic dynamics (which neglect the micromotion) can be described by an effective time-independent Hamiltonian $H^{\text {eff }}$. In the high frequency limit $\omega \gg h_{i}$, $V_{i}, \alpha_{i}, \beta_{i}, \gamma_{i}$, this effective Hamiltonian can be obtained by virtue of a Magnus expansion [44] (see Supplemental Material [45] for details). An expansion up to order $\mathcal{O}(1 / \sqrt{\omega})$ yields

$H^{\mathrm{eff}}=\sum_{i=1}^{L-2} \lambda_{i} \sigma_{i}^{z} \sigma_{i+1}^{x} \sigma_{i+2}^{z}+\sum_{i=1}^{L-1} V_{i} \sigma_{i}^{x} \sigma_{i+1}^{x}+\sum_{i=1}^{L} h_{i} \sigma_{i}^{x}$,

with coupling terms

$$
\lambda_{i, \text { odd }}=\cos \left(\phi_{0}\right) \alpha_{i} \beta_{i}, \quad \lambda_{i, \text { even }}=\cos \left(\phi_{0}\right) \gamma_{i} \alpha_{i+1} .
$$

The second and third terms in Eq. (3) arise from a firstorder Magnus expansion (which amounts to a time average) of $H_{2}$ (the first-order expansion of $H_{1}$ vanishes as the time average is zero). The first term in Eq. (3), which is an effective three-spin interaction, arises from the secondorder Magnus expansion that involves a time-averaged commutator of $\left[H_{1}(t), H_{1}\left(t^{\prime}\right)\right]$ (see Fig. 2 for a pictorial representation). All of the other commutators in the secondorder Magnus expansion, as well as all higher-order terms, scale away with $1 / \sqrt{\omega}$ or faster. We note that by this we have explicitly constructed an effective three-body Hamiltonian 


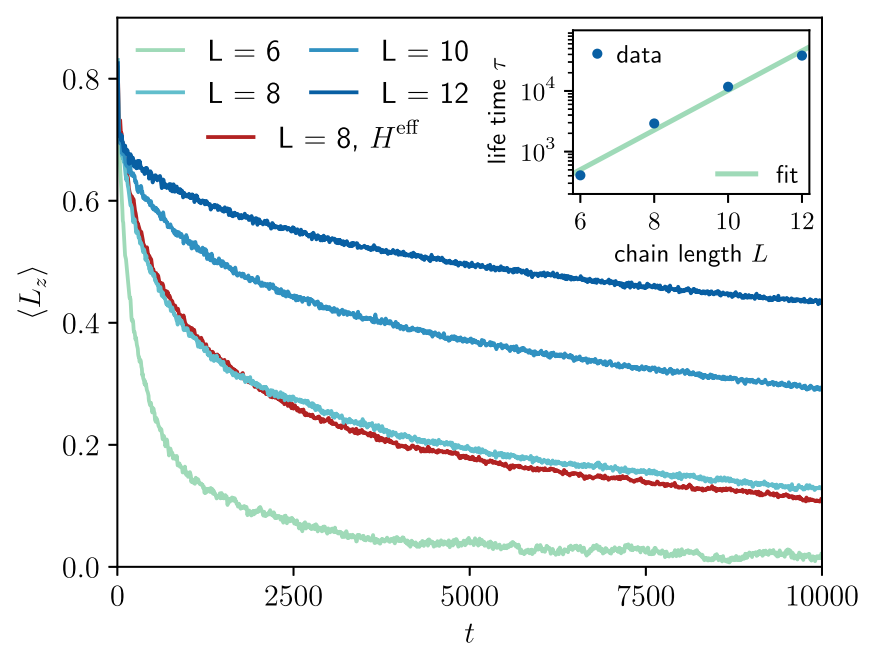

FIG. 3. Topological protection at high energies.-Time evolution of the edge spin $\left\langle L_{z}\right\rangle$ governed by Eqs. (1) and (2) for different system sizes and a driving frequency of $\omega=1000$. The edge spin decays exponentially with $\sim \exp [-\sqrt{t / \tau}]$. As the system size increases, the lifetime $\tau \sim \exp [0.752 L]$ of the spin becomes exponentially large in the system size (inset) due to the protection by topology and many-body localization even at high energies. The deviation from $\left\langle L_{z}\right\rangle=1$ is due to the fact that, although this operator has a nonzero overlap to the operator describing the topologically protected gapless edge mode, they are not identical. We choose $\phi_{0}=0$, disorder strengths $\sigma_{\alpha, \beta, \gamma}=1.0, \sigma_{V}=0.1, \sigma_{h}=0.05$, and average over 1000 random configurations. For $L=8$, we show data obtained using the effective time-independent Hamiltonian of Eq. (3) for comparison.

from a time-dependent two-body one. We choose to Floquet engineer this particular example of a three-body Hamiltonian as it has been shown before to harbor intriguing physics for which disorder coexists with symmetry protected topological features [39-42], which we will discuss in more detail in the "Results" section. However, one should note that this procedure is general and can be used to engineer effective three-body terms of a different desired form in the same way as illustrated here.

A benchmark of how well the Floquet-engineered physics of the time-dependent Hamiltonian given in Eqs. (1) and (2) agrees with that of the effective time-independent Hamiltonian $H^{\text {eff }}$ of Eq. (3) is shown in Fig. 3 as well as in the Supplemental Material [45]. We find perfect agreement in the high frequency limit where the Magnus expansion is justified. We emphasize that the results in this work are obtained using the full time-dependent Hamiltonian and that the effective Hamiltonian $H^{\text {eff }}$ only facilitates the physical interpretation.

Results.-The beauty of the effective Hamiltonian in Eq. (3) is that it can host a symmetry protected topological phase with protected gapless spin- $\frac{1}{2}$ edge excitations that can be used to define a qubit $[39,40]$. In the presence of disorder and interactions, many-body localization inhibits ergodicity and prevents the system from thermalizing, thereby extending the topological protection to high energies. The downside of this highly desirable behavior is that Eq. (3) contains three-body interactions that are generally not available. It is key to the understanding of the significance of the present scheme to acknowledge that no nearest-neighbor Hamiltonian $H=\sum_{i}\left(A_{i} B_{i+1}+\right.$ H.c. $)$ can give rise to the ground state of $H^{\mathrm{eff}}$ [46]. The Floquet approach presented here hence overcomes a roadblock against preparing such states of matter, as native three-body interactions of this type are generally not available. Cluster states as ground states of $H^{\text {eff }}$ can be approximated by nearest-neighbor Hamiltonians [47,48], but they require very strong interactions and high levels of control to effectively arrive at Hamiltonians with higher locality in perturbation theory.

Floquet engineering overcomes this hurdle [43] and moreover provides a natural way to dynamically control the edge qubit. Normally, a periodically driven system would heat up and eventually approach an infinitetemperature state. In our case, however, many-body localization suppresses heating. In a nutshell, the combination of Floquet engineering, topology, and many-body localization can be utilized to level disadvantages while harvesting the full advantages of the respective subfields. This allows one to implement and dynamically control a spin- $\frac{1}{2}$ qubit at high energies. We illustrate the three different aspects-disorder, heating, and control-separately in the following sections (see Fig. 1 for a schematic summary).

Topological protection.-The time-dependent Hamiltonian in Eqs. (1) and (2) hosts a symmetry protected topological phase at high energies since the system is barred from thermalization by many-body localization (in the sense that edge modes remain protected and suitable nonlocal order parameters take nonzero values even away from ground states). One can illustrate this explicitly by demonstrating that the gapless spin- $\frac{1}{2}$ edge mode has an infinitely long life. To this end, we prepare the system in a product state in which all the spins are initially pointing along the positive $z$ direction. The driving frequency is chosen as $\omega=1000$. We calculate the time evolution of the expectation value of the boundary spin $L_{z}=\sigma_{1}^{z}$ using the full $t \mapsto H(t)$ in Eqs. (1) and (2). The operator describing the exact topologically protected gapless spin- $-\frac{1}{2}$ edge mode at the left boundary has a nonzero overlap with $L_{z}$ and is of the form $\tilde{L}_{z}=a L_{z}+b B_{z}$ for suitable complex $a$ and $b$, with $B_{z}$ being a bulk contribution that does not contribute in the thermodynamic limit. The results are summarized in Fig. 3. As the system becomes larger, topological protection becomes increasingly robust, and the lifetime of $\left\langle L_{z}\right\rangle$ becomes exponentially large. We emphasize that the spins $\left\langle\sigma_{i}^{z}\right\rangle$ away from the edge decay quickly for arbitrary $L$ since they do not have a finite overlap with a topologically protected mode (data not shown). In a nutshell, Fig. 3 illustrates that topology and many-body localization team 


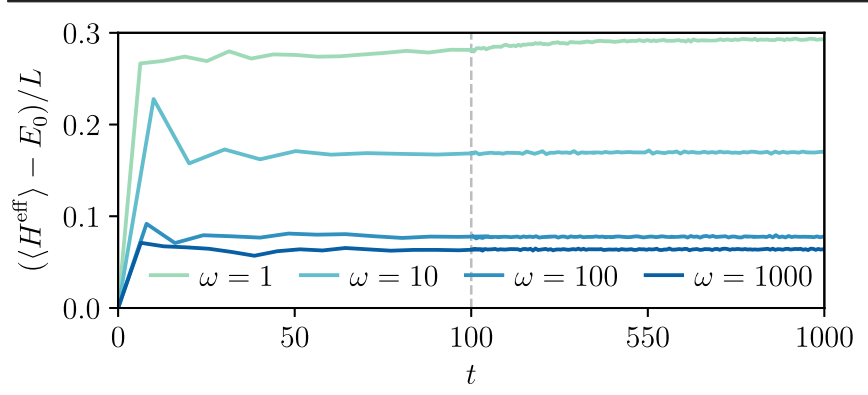

FIG. 4. Suppression of heating.- - Time evolution of the excess energy pumped into the system by the periodic drive. The energy saturates quickly, and the system does not approach an infinitetemperature state. This suppression of heating is due to manybody localization and detuning from single-particle resonances. We choose $L=10, \phi_{0}=0, \sigma_{\alpha, \beta, \gamma}=1.0, \sigma_{V}=0.1, \sigma_{h}=0.05$, and average over 1000 random configurations. Note that the scaling of the linear time axis changes at $t=100$.

up so that information can be stored robustly at the edge of a driven system at high energies.

Heating.-An interacting, periodically driven quantum system is generically expected to heat up over time [33]. This would be detrimental to our aim of storing information in the topologically protected edge states, as the system would heat up to infinite temperature, an equal superposition of all many-body states with no memory of the initial state. However, many-body localization bars the system from thermalizing [6,8], and it has been shown that this can be exploited to push the effects of heating to exponentially large times [34,35] where the scaling of the heating is determined by the localization length in the system. This is less obvious than it may seem, as manybody localized systems still allow for quantum information propagation [49], a feature that is reflected by a logarithmic entanglement growth in time following global quenches [50,51]. At the same time, an approximate emergent picture of $L$ quasilocal constants $C_{j}, j=1, \ldots, L$, of motion emerges for $H^{\text {eff }}$ that commute with each other and with $H^{\text {eff }}$. This suppression of driving-induced heating by the many-body localization can be understood by acknowledging that many-body localized systems behave very similarly to so-called integrable ones, which feature an extensive set of (quasi)local constants of the motion. These integrable systems are known to feature interesting periodically driven long-term states immune to runaway heating in contrast to generic (nonintegrable) driven systems [52].

Detuning away from single-particle resonances by going to high frequencies has a similar effect. Therefore, a system like ours is doubly protected from heating by employing both mechanisms: many-body localization and detuning. This is illustrated in Fig. 4, where we monitor the time evolution of the energy pumped into the system by the periodic drive in $t \mapsto H(t)$. We initially prepare the system in the ground state of the effective Hamiltonian in Eq. (3) of the corresponding Magnus expansion. The energy initially increases as the drive produces excess energy, which, if redistributed thermally, would translate to heating. As we approach the high frequency limit and Floquet engineer the many-body localized Hamiltonian in Eq. (3), excess heat production ceases and the system remains close to its ground state for arbitrary large times. This demonstrates that many-body localization and Floquet engineering conspire to efficiently suppress heating, which would be detrimental to storing information.

Control.-Finally, we demonstrate how to dynamically control the Floquet-engineered system. Our aim is to manipulate the edge spin on a short timescale by changing the parameters of the drive. To this end, we introduce a time-dependent phase $t \mapsto \phi(t)$ replacing $\phi_{0}$ in Eq. (1), which in general breaks the time periodicity. However, in either the case of (i), a slowly varying $\phi$, or (ii), a piecewise constant phase $\phi$, the above arguments still hold within each time interval in which $\phi$ is (approximately) constant. Changing the phase of the drive allows us to effectively control the system as the phase governs the magnitude of the couplings $\lambda_{i}$ in the effective Hamiltonian Eq. (3) via Eq. (4). We will now concentrate on the following, particularly instructive, example:

$$
\phi(t)= \begin{cases}0 & t<T_{1} \quad \text { or } \quad t>T_{2} \\ \pi / 2 & T_{1} \leq t \leq T_{2}\end{cases}
$$

We aim at rotating the spin and thus for times $T_{1} \leq t \leq T_{2}$ add a constant field along the $x$ direction, $H_{2} \mapsto H_{2}+$ $B_{x} \sum_{i} \sigma_{i}^{x}$. The effective (now piecewise time-dependent) Hamiltonian from a second-order Magnus expansion reads

$$
\begin{aligned}
H^{\mathrm{eff}}(t)= & \sum_{i=1}^{L-2} \lambda_{i}(t) \sigma_{i}^{z} \sigma_{i+1}^{x} \sigma_{i+2}^{z}+\sum_{i=1}^{L-1} V_{i} \sigma_{i}^{x} \sigma_{i+1}^{x} \\
& +\sum_{i=1}^{L}\left[B_{x}(t)+h_{i}\right] \sigma_{i}^{x},
\end{aligned}
$$

where

$\lambda_{i, \text { odd (even) }}(t)= \begin{cases}\alpha_{i} \beta_{i}\left(\gamma_{i} \alpha_{i+1}\right) & t<T_{1} \quad \text { or } \quad t>T_{2} \\ 0 & T_{1} \leq t \leq T_{2},\end{cases}$

and $B_{x}(t)=B_{x}$ for $T_{1} \leq t \leq T_{2}$.

In Fig. 5, we present results for the time evolution of the edge spin $\left\langle L_{z}\right\rangle$ for different switching times $T_{2}-T_{1} \sim 1$, $B_{x}=1.0$, and a driving frequency of $\omega=1000$ (disorder parameters are given in the caption). We again stress that the time evolution is calculated using the full timedependent Hamiltonian $t \mapsto H(t)$ and that its high frequency counterpart, the effective Hamiltonian $H^{\text {eff }}$, only facilitates the interpretation of the results. For times $t<T_{1}$, the edge state is protected by topology, and its information (i.e., being in the up state) can be stored for exponentially 

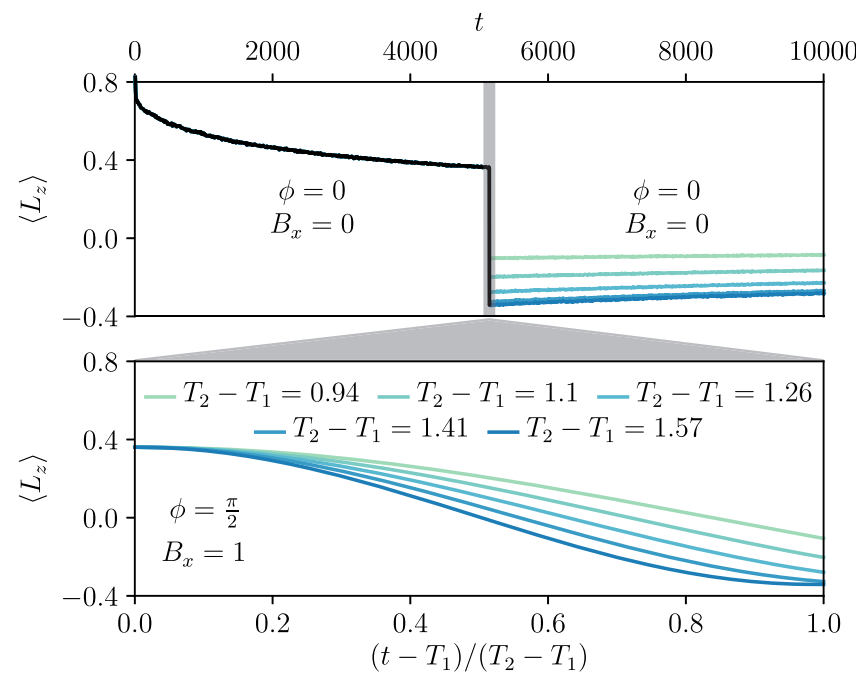

FIG. 5. Dynamical control.-The edge spin can be flipped on a short timescale $T_{1} \leq t \leq T_{2}, T_{2}-T_{1} \sim 1$ by applying a constant magnetic field $B_{x}$ as well as a brief $\pi / 2$ phase shift to the drive (lower panel). Before $\left(t<T_{1}\right)$ and after $\left(t>T_{2}\right)$, the spin is stable on exponentially long timescales (upper panel). We choose $L=10$, a driving frequency of $\omega=1000$, and disorder strengths $\sigma_{\alpha, \beta, \gamma}=1.0, \sigma_{V}=0.1$, and $\sigma_{h}=0.05$. Disorder averages are performed over 1265 random configurations.

long times. At $t=T_{1}$, the phase of the driven Hamiltonian is switched from $\phi_{0}=0$ to $\pi / 2$, and a magnetic field is applied. The magnetic field flips the direction of the spin. At time $t=T_{2}$, both the phase and the magnetic field are switched back off, $\phi_{0}=B_{x}=0$. The new state (where the edge spin now points down) can again be stored for exponentially long times as the topological properties are restored. This exemplifies how the versatility inherent to Floquet engineering can be utilized to dynamically control and manipulate the information in the topologically protected edge states.

Conclusion.-We have demonstrated how quantum states can be dynamically manipulated in a stable way in a periodically driven, realistic spin system. Starting from a simple spin model with two-body interactions, Floquet engineering allows one to design a toy Hamiltonian in which topology and disorder conspire to protect (qubit) spin states even away from low energies, as suggested in Refs. [39,40]. We explicitly demonstrated how heating is suppressed efficiently and investigated how well the toy Hamiltonian approximates the dynamics governed by the original one. Taking these properties together, this could provide a fascinating new route to implementing and controlling stable qubits, even at high temperatures, which should be the subject of future research.

This work has been supported by the Deutsche Forschungsgemeinschaft through the CRC 183 (Projects No. A01, No. A03, and No. B01), the DFG FOR 2724, the Cluster of Excellence Matter and Light for Quantum
Computing (ML4Q) EXC 2004/1-390534769, and through the Emmy Noether program (KA 3360/2-2). This work has also received funding from the European Union's Horizon2020 research and innovation programme under grant agreement No. 817482 (PASQuanS). We further acknowledge support from the Max PlanckNew York City Center for Non-Equilibrium Quantum Phenomena.

[1] A. Acin, I. Bloch, H. Buhrman, T. Calarco, C. Eichler, J. Eisert, D. Esteve, N. Gisin, S. J. Glaser, F. Jelezko, S. Kuhr, M. Lewenstein, M. F. Riedel, P. O. Schmidt, R. Thew, A. Wallraff, I. Walmsley, and F. K. Wilhelm, The quantum technologies roadmap: a European community view, New J. Phys. 20, 080201 (2018).

[2] T. D. Ladd, F. Jelezko, R. Laflamme, Y. Nakamura, C. Monroe, and J. L. O'Brien, Quantum computers, Nature (London) 464, 45 (2010).

[3] A. W. W. Ludwig, Topological phases: Classification of topological insulators and superconductors of non-interacting fermions, and beyond, Phys. Scr. T168, 014001 (2016).

[4] B. Zeng, X. Chen, D.-L. Zhou, and X.-G. Wen, Quantum Information Meets Quantum Matter: From Quantum Entanglement to Topological Phases of Many-Body Systems (Springer, Berlin, 2019).

[5] D. M. Basko, I. L. Aleiner, and B. L. Altshuler, Metalinsulator transition in a weakly interacting many-electron system with localized single-particle states, Ann. Phys. (Berlin) 321, 1126 (2006).

[6] R. Nandkishore and D. A. Huse, Many-body localization and thermalization in quantum statistical mechanics, Annu. Rev. Condens. Matter Phys. 6, 15 (2015).

[7] M. Schreiber, S. S. Hodgman, P. Bordia, H. P. Lüschen, M. H. Fischer, R. Vosk, E. Altman, U. Schneider, and I. Bloch, Observation of many-body localization of interacting fermions in a quasirandom optical lattice, Science 349, 842 (2015).

[8] D. A. Abanin, E. Altman, I. Bloch, and M. Serbyn, Colloquium: Many-body localization, thermalization, and entanglement, Rev. Mod. Phys. 91, 021001 (2019).

[9] D. A. Huse, R. Nandkishore, V. Oganesyan, A. Pal, and S. L. Sondhi, Localization-protected quantum order, Phys. Rev. B 88, 014206 (2013).

[10] A. Chandran, V. Khemani, C. R. Laumann, and S. L. Sondhi, Many-body localization and symmetry-protected topological order, Phys. Rev. B 89, 144201 (2014).

[11] A. Polkovnikov, K. Sengupta, A. Silva, and M. Vengalattore, Colloquium: Nonequilibrium dynamics of closed interacting quantum systems, Rev. Mod. Phys. 83, 863 (2011).

[12] J. Eisert, M. Friesdorf, and C. Gogolin, Quantum manybody systems out of equilibrium, Nat. Phys. 11, 124 (2015).

[13] A. Eckardt, Colloquium: Atomic quantum gases in periodically driven optical lattices, Rev. Mod. Phys. 89, 011004 (2017).

[14] V. Khemani, A. Lazarides, R. Moessner, and S. L. Sondhi, Phase Structure of Driven Quantum Systems, Phys. Rev. Lett. 116, 250401 (2016). 
[15] A. J. Friedman, R. Vasseur, A. C. Potter, and S. A. Parameswaran, Localization-protected order in spin chains with non-Abelian discrete symmetries, Phys. Rev. B 98, 064203 (2018).

[16] W. Berdanier, M. Kolodrubetz, S. A. Parameswaran, and R. Vasseur, Strong-disorder renormalization group for periodically driven systems, Phys. Rev. B 98, 174203 (2018).

[17] S. Roberts, B. Yoshida, A. Kubica, and S. D. Bartlett, Symmetry-protected topological order at nonzero temperature, Phys. Rev. A 96, 022306 (2017).

[18] Y. Kuno, Many-body localization induced protection of topological order in a XXZ spin model, Phys. Rev. Research 1, 032026 (2019).

[19] Z. Li, A. Chan, and T. B. Wahl, Classification of symmetryprotected topological phases in two-dimensional many body-localized systems, arXiv:1908.03928.

[20] P. Titum, E. Berg, M. S. Rudner, G. Refael, and N. H. Lindner, Anomalous Floquet-Anderson Insulator as a Nonadiabatic Quantized Charge Pump, Phys. Rev. X 6, 021013 (2016).

[21] F. Harper and R. Roy, Floquet Topological Order in Interacting Systems of Bosons and Fermions, Phys. Rev. Lett. 118, 115301 (2017).

[22] H. C. Po, L. Fidkowski, T. Morimoto, A. C. Potter, and A. Vishwanath, Chiral Floquet Phases of Many-Body Localized Bosons, Phys. Rev. X 6, 041070 (2016).

[23] H. Liu, T.-S. Xiong, W. Zhang, and J.-H. An, Floquet engineering of exotic topological phases in systems of cold atoms, Phys. Rev. A 100, 023622 (2019).

[24] B. Pérez-González, M. Bello, G. Platero, and A. Gómez-León, Simulation of 1D Topological Phases in Driven Quantum Dot Arrays, Phys. Rev. Lett. 123, 126401 (2019).

[25] D. J. Yates, F. H. L. Essler, and A. Mitra, Almost strong $(0, \pi)$ edge modes in clean interacting one-dimensional Floquet systems, Phys. Rev. B 99, 205419 (2019).

[26] N. H. Lindner, G. Refael, and V. Galitski, Floquet topological insulator in semiconductor quantum wells, Nat. Phys. 7, 490 (2011).

[27] M. A. Sentef, M. Claassen, A. F. Kemper, B. Moritz, T. Oka, J. K. Freericks, and T. P. Devereaux, Theory of Floquet band formation and local pseudospin textures in pumpprobe photoemission of graphene, Nat. Commun. 6, 7047 (2015).

[28] H. Hübener, M. A. Sentef, U. De Giovannini, A. F. Kemper, and A. Rubio, Creating stable Floquet-Weyl semimetals by laser-driving of 3D Dirac materials, Nat. Commun. 8, 13940 (2017).

[29] G. E. Topp, N. Tancogne-Dejean, A. F. Kemper, A. Rubio, and M. A. Sentef, All-optical nonequilibrium pathway to stabilizing magnetic Weyl semimetals in pyrochlore iridates, Nat. Commun. 9, 4452 (2018).

[30] A. G. Grushin, Á. Gómez-León, and T. Neupert, Floquet Fractional Chern Insulators, Phys. Rev. Lett. 112, 156801 (2014).

[31] M. Claassen, H. C. Jiang, B. Moritz, and T. P. Devereaux, Dynamical time-reversal symmetry breaking and photoinduced chiral spin liquids in frustrated Mott insulators, Nat. Commun. 8, 1192 (2017).
[32] D. M. Kennes, N. Müller, M. Pletyukhov, C. Weber, C. Bruder, F. Hassler, J. Klinovaja, D. Loss, and H. Schoeller, Chiral one-dimensional Floquet topological insulators beyond the rotating wave approximation, Phys. Rev. B 100, 041103(R) (2019).

[33] M. Bukov, L. D'Alessio, and A. Polkovnikov, Universal high-frequency behavior of periodically driven systems: From dynamical stabilization to Floquet engineering, Adv. Phys. 64, 139 (2015).

[34] P. Ponte, A. Chandran, Z. Papić, and D. A. Abanin, Periodically driven ergodic and many-body localized quantum systems, Ann. Phys. (Amsterdam) 353, 196 (2015).

[35] P. Bordia, H. Lüschen, U. Schneider, M. Knap, and I. Bloch, Periodically driving a many-body localized quantum system, Nat. Phys. 13, 460 (2017).

[36] O. Shtanko and R. Movassagh, Stability of Periodically Driven Topological Phases against Disorder, Phys. Rev. Lett. 121, 126803 (2018).

[37] S. Lieu, D. K. K. Lee, and J. Knolle, Disorder protected and induced local zero-modes in longer-range Kitaev chains, Phys. Rev. B 98, 134507 (2018).

[38] T. Orito, Y. Kuno, and I. Ichinose, Topological order versus many-body localization in periodically modulated spin chains, Phys. Rev. B 100, 214202 (2019).

[39] Y. Bahri, R. Vosk, E. Altman, and A. Vishwanath, Localization and topology protected quantum coherence at the edge of hot matter, Nat. Commun. 6, 7341 (2015).

[40] N. Y. Yao, C. R. Laumann, and A. Vishwanath, Manybody localization protected quantum state transfer, arXiv:1508.06995.

[41] K. S. C. Decker, D. M. Kennes, J. Eisert, and C. Karrasch, Entanglement and spectra in topological many-body localized phases, Phys. Rev. B 101, 014208 (2020).

[42] M. Goihl, N. Walk, J. Eisert, and N. Tarantino, Harnessing symmetry-protected topological order for quantum memories, Phys. Rev. Research 2, 013120 (2020).

[43] I.-D. Potirniche, A. C. Potter, M. Schleier-Smith, A. Vishwanath, and N. Y. Yao, Floquet Symmetry-Protected Topological Phases in Cold-Atom Systems, Phys. Rev. Lett. 119, 123601 (2017).

[44] W. Magnus, On the exponential solution of differential equations for a linear operator, Commun. Pure Appl. Math. 7, 649 (1954).

[45] See Supplemental Material at http://link.aps.org/ supplemental/10.1103/PhysRevLett.124.190601 for further information on the Magnus expansion, the dynamics found for a single disorder configuration as well as details about the numerical implementation.

[46] M. A. Nielsen, Cluster-state quantum computation, Rep. Math. Phys. 57, 147 (2006).

[47] S. D. Bartlett and T. Rudolph, Simple nearest-neighbor twobody Hamiltonian system for which the ground state is a universal resource for quantum computation, Phys. Rev. A 74, 040302(R) (2006).

[48] J. Kempe, A. Kitaev, and O. Regev, The complexity of the local Hamiltonian problem, SIAM J. Comput. 35, 1070 (2006). 
[49] M. Friesdorf, A. H. Werner, M. Goihl, J. Eisert, and W. Brown, Local constants of motion imply information propagation, New J. Phys. 17, 113054 (2015).

[50] M. Znidaric, T. Prosen, and P. Prelovsek, Many-body localization in the Heisenberg XXZ magnet in a random field, Phys. Rev. B 77, 064426 (2008).

[51] J.H. Bardarson, F. Pollmann, and J. E. Moore, Unbounded Growth of Entanglement in Models of
Many-Body Localization, Phys. Rev. Lett. 109, 017202 (2012).

[52] Outside of the many-body localized context we are discussing here exceptions to this rule where even integrable systems can heat up are known [53].

[53] T. Ishii, T. Kuwahara, T. Mori, and N. Hatano, Heating in Integrable Time-Periodic Systems, Phys. Rev. Lett. 120, 220602 (2018). 